\title{
RESULTADOS DE LA APLICACIÓN DEL PROCEDIMIENTO PARA LOCALIZAR PUNTOS DE VENTAS DEL MERCADO INTERNO EN LA AGENCIA VIAJES CUBANACÁN VILLA CLARA
}

\author{
Maité Rodriguez González \\ Universidad de la Habana \\ mrodriguez@ftur.uh.cu
}

\section{RESUMEN}

Los resultados de los estudios de localización de los puntos de ventas en las agencias de viajes ofrecen como principales beneficios ubicarlos en el lugar adecuado en correspondencia con las exigencias de los clientes. Aun cuando la tendencia actual en el sector turístico a nivel global es sustituir los puntos de ventas físicos por una distribución basada en las nuevas tecnologías, en Cuba estos estudios se hacen cada vez más imprescindibles, pues en la mayoría de los casos, se realizan basándose solamente en el empirismo. La agencia Viajes Cubanacán Villa Clara no está ajena a esta situación $\mathrm{y}$, a partir del auge de los productos destinados al mercado interno, requiere del análisis de las mejores ubicaciones de sus puntos de ventas a partir de la implementación de un procedimiento que se ajuste y adapte a sus particularidades y objeto de estudio. Es por ello que la siguiente investigación persigue identificar las mejores localizaciones de los puntos de ventas para el mercado interno en la agencia Viajes Cubanacán Villa Clara en aras de mejorar la gestión de su proceso de distribución,

PALABRAS CLAVE: Agencias de viajes, localización, puntos de ventas. 


\title{
RESULTS OF PROCEDURE TO FIND SALES POINTS OF THE INTERNAL MARKET IN THE TRAVEL AGENCY CUBANACÁN VILLA CLARA
}

\author{
Maité Rodriguez González \\ Universidad de la Habana \\ mrodriguez@ftur.uh.cu
}

\begin{abstract}
The results of studies about physical points of sales in travel agencies offer major benefits for these agencies in order to decide their locations based on customers requests. Although the global current trend in the tourism sector involves replacing physical points of sales due to the adoption of new technologies, in Cuba this has not occurred yet in the same magnitude. The agency Viajes Cubanacan Villa Clara is an example of this. The increase of tourism products for the domestic market requires the analysis of the best locations for sales. Thus, this article aims to identify the agency's best physical locations for the domestic market in order to improve its management of distribution process.
\end{abstract}

KEYWORDS: Travel agencies, location, sales points 


\section{Introducción}

La actividad turística en el destino Villa Clara ha experimentado durante los últimos años un crecimiento acelerado, dado principalmente por el desarrollo hotelero alcanzado en la cayería noreste y el impacto e implicación que han tenido las excusiones destinadas al mercado interno en la provincia, siendo estos una nueva medida, experimentada por el sector en los últimos períodos.

Estos productos surgen en el año 2009 como parte de una estrategia desplegada por el Ministerio de Turismo (MINTUR) a nivel nacional para hacerle frente a una fuerte demanda existente, por parte de los ciudadanos cubanos, de ofertas turísticas acorde a sus ingresos. Su finalidad fue la de incrementar los ingresos de las entidades turísticas cubanas, tanto a nivel nacional como territorial, a la vez que lograba la apertura de la venta de opcionales económicas a los turistas del mercado interno por parte de las agencias de viajes del país.

El surgimiento de las excursiones ha repercutido directamente en el desarrollo de las agencias de viajes, arrojando resultados alentadores, y las experiencias han sido gratificantes, a tal punto que, en la mayoría de las agencias, se han convertido en su producto líder. Todo ello ha provocado que las agencias de viajes del destino Villa Clara hayan tenido que enfrentar su repercusión, en aras de lograr una mejor forma de actuar y tomar decisiones.

En el caso específico de la agencia Viajes Cubanacán Villa Clara, a pesar de que se han obtenido resultados satisfactorios con la venta de estas opcionales, se ha detectado que, en los meses de julio, agosto septiembre, período de mayor demanda de los productos destinados al mercado interno, los resultados no son los deseados, pues se presentan ciertas deficiencias tales como la capacidad de venta de las excursiones en las agencias afectadas no es suficiente ante la demanda, provocando esto la aglomeración de clientes en la misma, y por consiguiente la pérdida de estos ante el insuficiente espacio del local; lo que se traduce en ventas perdidas, clientes desatendidos y servicios no prestados.

La solución no está en la gestión de los promotores de venta, quienes cuentan con un radio de acción limitado, sino en lograr atenuar el mercado que visita la agencia sin perderlo, por lo que se hace necesario entonces contar con puntos de ventas bien localizados para ofertar estos productos y que, además, actúen fuera de la propia entidad, lográndose con ello una mejor distribución de los productos con comodidad para el cliente y para la 
Se propone entonces identificar las mejores localizaciones de los puntos de ventas para el mercado interno en la agencia Viajes Cubanacán Villa Clara en aras de mejorar la gestión de su proceso de distribución

\section{Los puntos de ventas en las agencias de viajes}

El punto de venta, según Uribe (2012), es el lugar donde el cliente tiene contacto con personal que lo induce a la compra de un producto mediante técnicas adecuadas de persuasión. Si se aterriza dicha definición al sector turístico, el punto de venta es el lugar en el territorio nacional donde se oferta, al turista que arriba al país, los servicios que comercializa la organización, según plantea Mesa et al. (2005).

Si se tiene en cuenta que la AAVV puede consolidarse como un único centro de operaciones donde interviene uno o más puntos para efectuar la venta de servicios o productos turísticos, o, por el contrario, como una cadena de locales o puntos de ventas repartidos por una zona determinada, se puede identificar a sus puntos de ventas como aquel espacio físico que permite ofrecer y vender productos y servicios turísticos a los clientes que lo demanden, brindando información necesaria y posibilitando la realización de reservas y venta de servicios y productos turísticos por anticipado. La implementación de un sistema de puntos de venta en las AAVV no es un lujo, sino una necesidad primordial para agilizar los procesos, aprovechar oportunidades y cubrir necesidades.

\section{Antecedentes}

A pesar de la importancia que tienen los puntos de ventas en las AAVV, las últimas tendencias del sector turístico plantean que el papel y actividad de los puntos de ventas en estas intermediarias desciende y descenderá en el futuro a nivel mundial, siendo esto el resultado, según criterio de los autores De Borja y Miquel (2009), de la presencia de avances tecnológicos y cambios sociales y económicos. Esta situación en el destino Cuba dista mucho de ser semejante a lo que ocurre en la orbe, y se debe, específicamente, a que debido a las dificultades en el acceso a las nuevas tecnologías de la información y las comunicaciones (TIC), sobre todo a Internet, que tiene el país, ha provocado que los puntos de ventas físicos cerca de los lugares de residencia continúen siendo la salida fundamental para que las personas adquieran servicios y productos turísticos.

Es por ello que, a partir del auge de las demandas que han tenido algunos productos en el mercado interno, tales como las excursiones destinadas a este mercado, las agencias 
de viajes tienen como necesidad imperante establecer puntos de ventas bien localizados para ofertar estos productos, logrando así que estos lleguen al cliente adecuado, en el momento preciso y se oferten en el lugar idóneo, permitiendo ello acceder al mercado objetivo, acercar el producto turístico al mismo propiciando su compra y satisfacer la solicitud creciente por parte de los clientes.

La agencia Viajes Cubanacán Villa Clara no está exenta a esta situación y sigue prestándole especial atención a la implementación de los puntos de ventas físicos para ofertar sus servicios y productos turísticos, y más aún cuando es evidente el auge de los excursiones destinadas a los cliente nacionales, mercado que figura como uno de los principales en la agencia. Dicha implementación requiere de estudios de localización, los cuales son imprescindibles y esenciales para las entidades que necesiten crear o mover determinado punto de venta. Sin embargo, a nivel global, estos estudios han visto frenado su avance con la aparición de las TIC, cediendo importancia a dicho análisis. Los investigadores del sector, le prestan mayor atención al papel de Internet en la distribución turística que a los puntos de ventas físicos y los estudios asociados a su implementación. Todo ello, unido a las dificultades presentadas por los clientes para acceder a las TIC, sobre todo a Internet, ha provocado mayor interés de la agencia de viajes por realizar una correcta localización de sus puntos de ventas, que debe estar basada en la aplicación de metodologías o procedimientos específicos para este tipo de investigación. Sin embargo, en la consulta bibliográfica realizada, evidencia la insuficiencia de literatura en lo referente a la existencia de procedimientos o metodologías, adecuados al tema de investigación y a las agencias de viajes, las cuales presentan características muy peculiares debido a los servicios y productos que ofertan.

Solo se encontró un procedimiento para realizar la ubicación de los puntos de ventas en agencias de viajes, las demás propuestas de metodologías y procedimientos a seguir se llevan a cabo para localizar las instalaciones de manera general y enfocadas a las decisiones de localización basadas en el emplazamiento del local, por lo que tratan, de manera aislada, el enfoque al cliente como eje primordial en las decisiones de localización de puntos de ventas para estas intermediarias.

Además en la agencia se carece de instrumentos necesarios para llevar a cabo de manera eficiente el proceso de localización de sus puntos de ventas. Al igual que sus competidoras reales, las agencias Cubatur, Gaviota Tours, Havanatur y Ecotur, realiza el proceso de localización de sus establecimientos de manera empírica, o sea basado en las experiencias adquiridas por los trabajadores y directivos en el proceso de comercialización de los productos destinados al mercado interno. 


\section{Metodologías usadas}

Para el desarrollo de la investigación se emplearon métodos del nivel teórico tales como histórico - lógico, modelación, análisis-síntesis e inducción-deducción; del nivel empírico el análisis de documentos y la técnica Tormenta de ideas; métodos estadísticos y algunas técnicas para la recopilación de datos como las entrevistas y encuestas a clientes, directivos y trabajadores.

Se emplearon técnicas que para realizar la segmentación de los mercados de las agencias tales como el método de Belson, el análisis de la varianza, la Chi-cuadrado, el método de Sonnsquist y Morgan, el modelo de Canguilhem y el análisis discriminante.

Para determinar la metodología a utilizar se analizaron cinco propuestas para localizar puntos de ventas encontradas en la revisión bibliográfica, siendo estas:

- Metodología para la localización de puntos de ventas (Núñez, 2001).

- Procedimiento general para la toma de decisiones de localización (García-Sabater, 2004).

- Procedimiento para la localización de un establecimiento (Kotler et al., 2004).

- Metodología para identificar el mejor lugar para ubicar estratégicamente un negocio o una red de puntos de ventas (Bogotá Emprende, 2010).

- Procedimiento para localizar los puntos de ventas para el mercado interno en las agencias de viajes (Rodriguez, 2013) (Ver Anexo 1).

Del análisis comparativo realizado por la autora se demostró que de los procedimientos consultados referentes a la localización de instalaciones sólo el de Rodriguez (2013) se adapta a los objetivos de la investigación, siendo este además el que reúne la mayoría de los criterios incluidos en el análisis, seguido por el procedimiento de Kotler et al. (2004), empleando 8 criterios en su propuesta, lo que representa un $73 \%$ del total. Con respecto al resto de los autores, García-Sabater (2004) sólo utiliza 5 de estos criterios, coincidiendo con este Bogotá Emprende (2010), representando cada uno el 46\% del total. Mientras que Núñez (2001) representa el 37\% del total de criterios, pues utiliza sólo 4 de estos criterios en su propuesta. De acuerdo al objetivo de la investigación, los procedimientos de García-Sabater (2004), Bogotá Emprende (2010), Núñez (2001) no son aplicados en las entidades objeto de estudio, sólo Kotler et al. (2004) y Rodriguez (2013) es utilizado en entidades turísticas, el primero se emplea fundamentalmente en 
hoteles y restaurantes, mientras que en el segundo es específico para las agencias de viajes. Siendo esta la causa por lo que se aplicó como método principal el procedimiento de Rodriguez (2013), el cual reúne los requisitos necesarios para determinar la ubicación idónea de los puntos de venta en las agencias, además de ajustarse a la situación concreta de las agencias, a sus características particulares, respondiendo a sus requerimientos y prevaleciendo el enfoque al mercado al que se dirige la oferta. Por tanto, constituye una poderosa arma para decidir y justificar la adecuada localización de puntos de ventas de la agencia objeto de estudio, sobre la base de un fundamento teórico y lógico.

\section{Situación actual del estudio para localizar los puntos de ventas para el mercado interno en la agencia Viajes Cubanacán Villa Clara}

A partir del año 2011, como consecuencia del auge que ha tenido la venta de los productos destinados al mercado interno, la agencia Viajes Cubanacán incorporó en su grupo de Estrategias Comerciales la creación de puntos de venta móviles para este producto, siendo el principal objetivo de la estrategia, acercar el producto a los clientes durante la temporada alta de operación de la opcional, aunque cabe la posibilidad de que algunos puntos continúen su operación durante el resto del año, todo depende de la demanda en el lugar donde se ubique el mismo. No obstante a ello, las decisiones de localización de estos puntos de ventas no se encuentran justificadas mediante estudios previos; la decisión de apertura o cierre de determinado punto de venta es emitida en el Consejo de Dirección de cada agencia por iniciativa propia o por experiencia de otras agencias.

Se puede concluir entonces que existe la necesidad de implementar un procedimiento que permita la localización de los puntos de venta para el mercado interno en la agencia de viajes, encaminado a lograr la gestión efectiva del proceso de venta y distribución de dicho producto en la intermediaria objeto de estudio; con un enfoque al cliente y orientado a la transformación y mejora continua de los resultados, todo ello en función de satisfacer las exigencias de los clientes y hacer frente a la competencia.

\section{Procedimiento para localizar los puntos de ventas para el mercado interno en la agencia Viajes Cubanacán Villa Clara}

\subsection{Descripción en detalle de cada una de las fases y etapas que se proponen en el procedimiento}

Fase 1. Analítica: Diagnóstico del proceso de distribución en una agencia de viajes.

Etapa 1. Selección y entrenamiento de los miembros del equipo de trabajo: se conformó el equipo de trabajo interdisciplinario, siendo el máximo responsable del estudio el director de la agencia de viajes. Se definieron otros 6 integrantes los cuales relacionan con el área 
de gestión a estudiar, con experiencias en las actividades incluidas en la investigación y con conocimientos del proceso de trabajo de la agencia, de manera general. Se entrenó el equipo de trabajo explicándole la investigación a desarrollar, su objetivo, técnicas y métodos a emplear para ello.

Etapa 2. Análisis externo: se realizó un estudio de las demandas del entorno de la agencia de viajes, centrando su investigación en el análisis en los factores económico, social-cultural, político-legal y tecnológico. Se concretaron los principales clientes, proveedores o prestatarios, competidores existentes, competidores potenciales y productos sustitutivos. Se incluyó además un estudio del proceso de distribución del destino y una breve caracterización de las tendencias actuales de los mercados del destino en cuestión. El análisis culminó con la fijación de las principales Oportunidades y Amenazas.

Etapa 3. Análisis interno: se inició con una caracterización del proceso de distribución en la agencia de viajes donde se detallaron las particularidades del proceso de distribución en la agencia, se explicaron sus etapasy los servicios que incluye, se esquematizó la red de distribución utilizada por la agencia basada en la integración de los diferentes canales de distribución con que opera para hacer llegar sus servicios y productos turísticos al consumidor final y, por último, se definieron las relaciones internas de las entidades que integran los canales de distribución definidos. Se realizó un análisis del producto, precio, comunicación y ventas y de sus principales puntos de ventas, se evaluó lainserción, en el proceso de distribución, de las Nuevas Tecnologías de la Información y las Comunicaciones (TIC), se analizó losrecursos económicos, humanos y materiales para desarrollar la distribución. Por último, se definieron las principales Debilidades y Fortalezas de la agencia de viajes analizada.

Etapa 4. Análisis DAFO: se realizó un análisis de los resultados obtenidos en las fases y etapas anteriores, utilizando el estudio matricial DAFO, exponiendo la situación actual del proceso de distribución del producto TEC en la agencia de viajes objeto de estudio.

Fase 2. Estratégica: Estudio de las excursiones económicas en la agencia de viajes: se refirió a la búsqueda de información para establecer posibles alternativas de localización.

Etapa 5. Caracterización y comportamiento de las excursiones destinadas al mercado interno en la agencia de viajes: se realizó un análisis de las características principales del producto TEC, dando a conocer su definición, surgimiento, particularidades y comportamiento en la AAVV estudiada.

Etapa 6. Determinación de los segmentos de mercados de la AAVV: se estudió el mercado objetivo de la agencia, para lo cual se identificó cada uno de los segmentospara 
orientar adecuadamente la oferta a cada uno de ellos.

Etapa 7. Análisis de la competencia de la AAVV: se detalló la situación actual del producto TEC en las principales agencias de viajes que conforman la competencia, analizando su comportamiento durante los últimos años, sus principales productos, segmentos de mercados y puntos de ventas para vender el producto TEC, prescribiendo las acciones estratégicas empleadas para localizarlos.

Fase 3. Operativa: Determinar los puntos de ventas a implementar: se establecieron los puntos de ventas necesarios en la agencia de viajes.

Etapa 8. Definir principal (es) mercado (s) objetivo (s) de la agencia de viajes: se concretaron los principales mercados objetivos de la agencia, seleccionando aquellos segmentos que resulten más adecuados para el logro de la estrategia comercial de la agencia. Se utilizaron tres criterios, según Serra (2003), para establecer la prioridad de los segmentos: el tamaño y potencial de crecimiento del segmento, El atractivo estructural del segmento y los objetivos y recursos de la empresa o del destino.

Etapa 9. Definir áreas geográficas para abrir los puntos de venta: se fijaron las áreas geográficas propicias para abrir los nuevos establecimientos, teniendo en cuenta las área/s de mercado actual/es y potencial/es por municipios. Estas áreas serán establecidas en correspondencia con los lugares donde se encuentra el principal (es) mercado (s) objetivo (s) de la agencia de viajes, identificados estos en la etapa 8.

Etapa 10. Definir cantidad de puntos de ventas: se precisó el número de puntos de ventas que serán necesarios implementar para hacerle frente a la demanda existente en la agencia. El análisis se efectuó teniendo en cuenta las estrategias, políticas y proyecciones futuras de la entidad.

Etapa 11. Determinar principales características de los puntos de ventas a implementar por la agencia de viajes: se decidieron las características a reunir por estos puntos, explicándose su funcionamiento basado en las estrategias comerciales a seguir por cada agencia. Se analizó si en el área geográfica donde se abrirá el punto de ventas existen establecimientos pertenecientes al sector o a la cadena u otro organismo donde se puede habilitar un local para la ubicación del punto de venta y si este local será arrendado o comprado. Se analizó la tipología del punto de venta a implementar teniendo en cuenta frecuencia de prestación del servicio, o sea, si para la agencia es más factible abrir un punto de venta móvil o fijo en determinada área geográfica. Se estableció horario de apertura y cierre así como la frecuencia semanal de funcionamiento. Se especificó el objetivo que persigue la apertura del punto de venta en el lugar señalado y la cantidad de personas que laborarán en el nuevo establecimiento. 
Etapa 12. Búsqueda de posibles localizaciones para implementar los puntos de ventas: se precisaron, a través de una propuesta, las posibles alternativas de localización de los puntos de ventas, para lo cual se utilizó la técnica conocida como Lluvia de Ideas.

Etapa 13. Definir los criterios decisionales: se establecieron, para evaluar la propuesta del conjunto de localizaciones candidatas desarrollada en la etapa anterior, un conjunto de criterios de decisión a tener en cuenta en el proceso de localización de los puntos de ventas, los cuales se enumeran a continuación:

1. La prefactibilidad económica, la cual incluye la prefactibilidad técnica, organizacional y financiera del punto de venta.

2. La población.

3. La accesibilidad al punto de venta.

4. La afluencia de personas al lugar donde se ubica el punto de venta.

5. La visibilidad del punto de venta.

6. La cercanía a los competidores.

7. Las condiciones de la infraestructura de los puntos de ventas.

8. La disposición de las entidades a brindar su apoyo para implementar el punto de venta.

Etapa 14. Evaluar las alternativas de localización propuestas: se procedió a realizar un análisis detallado de cada una de las alternativas propuestas en la etapa 12. Para ello se aplicó la técnica Factores Ponderados, la cual, según García-Sabater (2004) permite asignar valores cuantitativos a todos los factores relacionados con cada alternativa de decisión y derivar una calificación compuesta que puede ser usada con fines de comparación.

Etapa 15. Selección de la (s) mejor (es) alternativas para localizar los puntos de ventas: una vez evaluadas las alternativas de localización se compararon entre sí para conseguir determinar una o varias localizaciones idóneas para la agencia de viajes. Además el equipo de trabajo tuvo en cuenta otros factores subjetivos para determinar la localización definitiva de los puntos de ventas.

\subsection{Principales resultados de la aplicación del procedimiento para localizar los puntos de ventas para el mercado interno en la agencia Viajes Cubanacán Villa Clara}

1. Se determinó como solución estratégica que, si se atenúa la carencia de suficientes puntos de ventas para responder al crecimiento acelerado de la demanda de los productos destinados al mercado interno durante los meses julio, agosto y septiembre, la agencia estará en mejores condiciones de enfrentar el fortalecimiento de los principales competidores del destino. 
2. La demanda de los productos destinados al mercado interno ha experimentado un crecimiento considerable desde que la agencia comenzó en el año 2009 a incluirlo en su catálogo de ofertas, superando los registros históricos de ingresos obtenidos por ventas en buró y convirtiéndose en su producto líder (Ver gráfico 1).

Gráfico 1. Comportamiento de la demanda del producto TEC en la agencia Viajes Cubanacán Villa Clara.

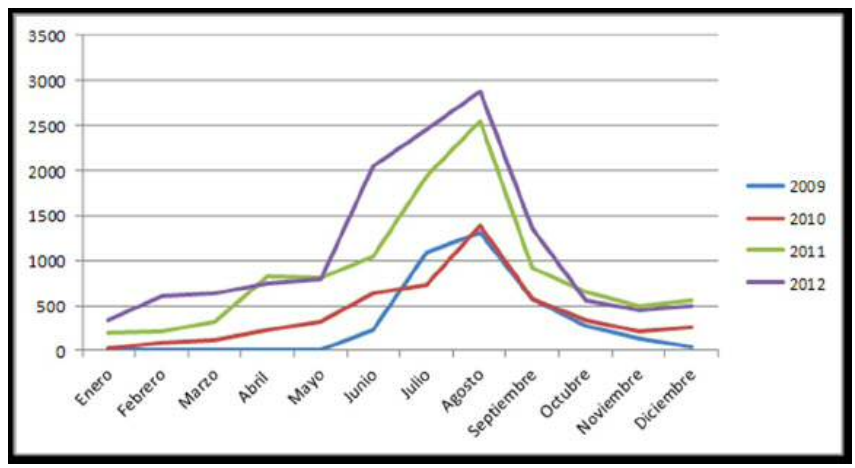

Fuente: Elaboración propia

3. El destino turístico más vendido por la agencia fue Varadero y el hotel Villa la Mar, situado en este mismo destino. El mayor número de ventas se realizó en el buró de venta de la agencia con un $78 \%$, seguido por sus puntos de ventas Islazul, Infotur y Praga respectivamente.

4. Los clientes que más demandan en la agencia los productos para el mercado interno son los nacionales, quedando en un segundo plano el mercado convencional o extranjero, el cual se compone por los clientes que se encuentran de recorrido por la provincia; los hospedados en casas particulares, que por lo general son provenientes de Francia, Inglaterra, Canadá e Italia; los estudiantes de la Universidad Central "Marta Abreu" de Las Villas y de la Universidad de Ciencias Médicas, en su mayoría provenientes de Sudáfrica y Argentina, y por último, los cubanos residentes en el extranjero, principalmente en los Estados Unidos de América.

5. El análisis de la encuesta aplicada al mercado nacional demostró que el $42 \%$ del total de clientes encuestados reside en la ciudad de Santa Clara, el 19\% en Caibarién, el $15 \%$ en Placetas, el 12\% en Sagua la Grande y el 12\% restante agrupa a clientes residentes en Remedios, Camajuaní, Santo Domingo y Manicaragua; la edad de la mayoría de los clientes que compran en la agencia oscila entre 36 y 65 años, siendo el número de 
mujeres superior al de hombres; el $40 \%$ de los encuestados tiene nivel universitario, el $34 \%$ técnico, el $21 \%$ medio o secundario y el $5 \%$ restante, básico; pero no todos están vinculados a un centro laboral; el 59\% tiene un nivel de ingresos altos, el 31\% medio y el $10 \%$ bajo; el $46 \%$ viaja acompañado de 2 a 5 personas y el $38 \%$ con una persona; el $54 \%$ de los encuestados manifestó viajar con sus familiares, el $31 \%$ con su pareja y el $15 \%$ con amigos; la mayoría manifestó que compra en la agencia una vez al año y generalmente en el tercer trimestre.

6. Se establecieron ocho segmentos de mercados principales, los cuales son grupos de consumidores relativamente homogéneos gracias al arreglo de la variable procedencia con las demás variables utilizadas en la encuesta.

7. La operación de los productos destinados al mercado interno en las agencias del destino, que forman parte de la competencia de Viajes Cubanacán, ha crecido de forma alarmante, siendo la agencia Cubatur quien logró mover la mayor cantidad de pax durante el 2010, pero esto no se tradujo en los mayores ingresos. En los años 2010 y 2011 las agencias Gaviota Tours y Havanatur han experimentado un salto cuantitativo considerable en sus cifras, mostrando un crecimiento acelerado de sus ingresos, no siendo así para Cubatur, que muestra un menor por ciento de crecimiento (Ver tabla 1).

Tabla 1. Análisis comparativo de la competencia.

\begin{tabular}{|c|c|c|c|c|c|}
\hline \multirow{2}{*}{ Agencias } & \multicolumn{2}{|c|}{ Pax } & \multicolumn{2}{c|}{ Ingresos } & $\begin{array}{c}\text { \% de } \\
\text { crecimiento }\end{array}$ \\
\cline { 2 - 7 } & 2010 & 2011 & 2010 & 2011 \\
\hline Cubanacán & 5778 & 10501 & 545014.20 & 724524.00 & 32 \\
\hline Cubatur & 8471 & 9185 & 371700.40 & 483623.22 & 30 \\
\hline Havanatur & 1421 & 4278 & 105349.33 & 296230.00 & 81 \\
\hline Gaviota Tours & 799 & 3068 & 76304.45 & 226537.53 & 97 \\
\hline
\end{tabular}

Fuente: Elaboración propia

8. Los catálogos de ofertas de las AAVV que conforman la competencia son muy similares, siendo la única diferencia el factor precio, el cual varía en dependencia del margen de beneficio que cada agencia fija al precio de sus productos.

9. Se determinaron como principales mercados objetivos los ubicados en los municipios de Santa Clara, Caibarién, Placetas y Sagua la Grande, definiéndose además una cantidad de cinco puntos de ventas para distribuirlos entre ellos: dos puntos en el municipio de Santa Clara y un punto en Placetas, otro en Caibarién y uno en Sagua la Grande. 
10. Se establecieron como principales características de los nuevos puntos de ventas a implementar que estos no serán fijos sino puntos de ventas móviles, pues se abrirán solo durante los meses de mayor demanda de los productos destinados al mercado interno, del 1ro de julio al 30 de septiembre; los buroes de venta radicados en los municipios Placetas, Caibarién y Sagua la Grande se alternarán, prestando servicios dos días en la semana, mientras que los dos puntos a localizar en la ciudad de Santa Clara, también se alternarán, pero prestarán servicios durante tres días en la semana; los locales para ubicar los puntos no serán arrendados ni comprados, será una prestación de locales de las entidades pertenecientes al Ministerio del Turismo (MINTUR).

11. Se realizó una búsqueda de posibles localizaciones para implementar los nuevos puntos de ventas, estableciéndose un conjunto de localizaciones candidatas en las entidades pertenecientes al MINTUR situadas en cada una de las áreas geográficas consideradas claves (Ver anexo 2).

12. Se definieron como criterios decisionales a utilizar en la evaluación de las diferentes alternativas la afluencia de personas al lugar, la accesibilidad y visibilidad, las condiciones de la infraestructura, la cercanía a los competidores, la disposición de acogida de las entidades y la población. Se incluyen además en su lista de criterios la ubicación geográfica y el horario de prestación de servicios, por constituir estos, según su opinión, de relevante importancia para el desarrollo efectivo de la investigación en la agencia.

13. Se evaluaron las alternativas de localización existentes, mediante el método Factores Ponderados de García-Sabater (2004), dando como resultado que en la ciudad de Santa Clara los puntos de venta se ubicarían en el Ditú Riviera y en el Ditú Hospital, en Placetas estaría en la Cafetería "Café Parque", en Caibarién se ubicaría en la Cafetería "Villa Blanca", mientras que la alternativa de localización para Sagua la Grandes sería la Cafetería "Gran Rey".

14. Posterior al estudio realizado la agencia Viajes Cubanacán implementó puntos de ventas en los municipios de Placetas, Camajuaní y Remedios, funcionando hasta la fecha el ubicado en Placetas, el resto fueron cerrados por no obtener los resultados esperados con su apertura. Todo lo cual demuestra que solo el punto de venta ubicado en Placetas, municipio que fue seleccionado en la investigación realizada con anterioridad, no fracasó a la implementación. Camajuaní y Remedios, no se incluyeron en los municipios propuestos para implementar los puntos de venta, de ahí que los resultados obtenidos no fueron catalogados como satisfactorios. 


\section{Conclusiones}

1. La agencia Viajes Cubanacán presenta, en la actualidad, una correcta implementación de sus puntos de venta como guía para lograr mejorar su canal de distribución e incrementar las ventas y los niveles de satisfacción de sus clientes.

2. La evaluación del estudio de localización de los puntos de ventas para el mercado interno en la agencia Viajes Cubanacán Villa Clara comprueba que no se han realizado estudios previos para localizar sus puntos de venta, de modo que la implementación se ha desarrollado de forma espontánea y empírica, lo que les impide la realización de una gestión de la distribución orientada a satisfacer los deseos del cliente.

3. Se encontraron propuestas de procedimientos y metodologías, realizadas por diferentes autores, para lograr la localización de instalaciones, referidas a estas de manera general y solo dos a empresas turísticas. La mayoría de ellas están enfocadas a las decisiones de localización basadas en el emplazamiento del local, solo la propuesta de Rodriguez (2013) trata el enfoque al cliente como eje primordial en las decisiones de localización de puntos de ventas en las agencias de viajes.

4. El procedimiento aplicado en la agencia Viajes Cubanacán Villa Clara se desglosa en 3 fases y 15 etapas las cuales tratan los puntos generales que debe contener todo proceso decisional de localización. Además responde a las necesidades actuales de la agencia, pues le permitió contar con un instrumento metodológico para perfeccionar el proceso de distribución del producto destinado al mercado interno en la agencia y, por tanto, lograr la localización idónea de sus puntos de venta.

\section{Bibliografía}

Abreu, M. (2011). Indicación no 01/2011 "sobre apertura y cierre de buroes de turismo". Casa Matriz Viajes Cubanacán. La Habana.

Acerenza, M. Á. (1997). Agencias de viajes. Organización y operación, México.

Álcazar, B. D. (2002) Los canales de distribución en el sector turístico, Madrid, España. Ayala, H.; Martín, R.; Rodríguez, R. (2003). Organización económica y gestión de entidades participantes en el turismo, Ciudad de la Habana. Cuba.

Borja, L. D.; Miquel, J. (2009). Nuevo paradigma de la intermediación turística. Colección Turismo. Pirámide Ediciones. Madrid.

De Arquer M. I. (2006). “Fiabilidad humana. Métodos.” Centro nacional de condiciones de trabajo. Disponible en: www.mtas.es/insht/ntp/ntp

Díaz, M. M. A.; Jouve, M. C.; Linares, L. A. (2008). Material de agencias de viajes. Escuela de Altos Estudios de Hotelería y Turismo. La Habana, Cuba.

Diéguez, E. L.; Pérez, P. A. (2007). Métodos de localización de instalaciones de producción y servicios. Universidad de Matanzas Camilo Cienfuegos. Cuba.

Escalona, R.; Batista, C. (2011). "Perfil de los turistas nacionales del mercado interno 
en la agencia de viajes Cubanacán Holguín.” Disponible en: http://www.eumed.net/rev/ turydes/11/ebbm.html

García-Sabater, J. P. (2004). Diseño de sistemas productivos y logísticos. Localización de instalaciones. Universidad Politécnica de Valencia. España.

González, O. (2005). Papel de la localización en la selección de establecimiento minorista.

Jáuregui, A. (2001). "Localización \& criterio invisible de mercadeo". Disponible en: http://www.gestiopolis.com

Kotler, P.; Bowen, J.; Makens, J. (2004). Marketing para turismo, Madrid, España.

Matos, J. A. (2008). "La actuación de las agencias de viajes receptivas en Cuba". Disponible en: http://www.monografias.com

Rodriguez, M. (2013). Procedimiento para localizar los puntos de ventas del producto TEC en la agencia de viajes del destino Villa Clara. Tesis presentada en opción al Título Académico de Máster en Gestión Turística. Centro de Estudios Turísticos. Universidad Central "Marta Abreu" de Las Villas. Santa Clara. Cuba.

Serra, A. (2003). Marketing turístico. IN ESIC EDITORIAL. EDICIONES PIRÁMIDE (GRUPO ANAYA, S. A. (Ed.) Madrid, España.

Vigaray, D. J. (2004). Comercialización y retailing: Distribución comercial aplicada. Ed Pearson Educación-Prentice Hall. ISBN: 84-205-4372-1 